\title{
A mobile learning system for scaffolding bird watching learning
}

\author{
Y.S. Chen, T.C. Kao \& J.P. Sheu \\ National Chung Cheng, National Dong Hwa \& National Central University, Taiwan
}

\begin{abstract}
This paper develops a mobile learning system for scaffolding students learning about bird-watching. The aim is to construct an outdoor mobile-learning activity using up-to-date wireless technology. The proposed Bird-Watching Learning (BWL) system is designed using a wireless mobile ad-hoc network. In the BWL system, each learner has a PDA (Personal Digital Assistant) with a Wi-Fi-based (IEEE 802.11b) wireless network card. The BWL system offers a mobile learning system which supports the students learning through scaffolding. The aim of a formative evaluation was twofold: to explore the possible roles and scaffolding aids that the mobile learning device offers for bird-watching activities and to investigate whether student learning benefited from the mobility, portability, and individualisation of the mobile learning device.
\end{abstract}

Keywords: Bird-watching; Formative; Intranet; Mobile; Quantitative; Scaffolding; School; Wireless

\section{Introduction}

Advances in wireless communication technologies have recently provided the opportunity for educators to create new educational models (Johnson \& Maltz, 1996; Lin \& Liu, 1999; Chen \& Nahrstedt, 2000; Chen \& Lai, 2001). With the aid of wireless communication technology, educational practice can be embedded into mobile life without wired-based communication. With the trend of the educational media becoming more mobilised, portable, and individualised, the learning form is being changed dramatically. This work aims to synthesise the cognition and technology domains to establish a new outdoor-ecological learning model. The mobile learning environment posses many unique characteristics, they are:

- Urgency of learning need: The wireless applications can be used for an urgent matter of learning, such as linking problem solving and knowledge. Otherwise, the learner may record the questions and look for the answer later in the library, on line with a computer, or from the experts.

- Initiative of knowledge acquisition: Most of the time, information provided by wireless applications is based on the learners' requests, i.e. information on demand. Being based on the learners' request, the wireless application can provide closely related information in time.

- Mobility of learning setting: The wireless devices are developed to be more and

Accepted 15 April 2003

Correspondence: Yuh-Shyan Chen, Department of Computer Science and Information Engineering, National Chung Cheng University, Chiayi. Taiwan, R.O.C. Email: yschen@cs.ccu.edu.tw 


\section{Y.S. Chen et al.}

more portable. Therefore, the educational practice can be performed at any time and any place, such as on a tour bus, camping area, exhibit room, etc. All kinds of field trip situations can be facilitated. This kind of learning setting can be preplanned or be opportunistic in nature.

- Interactivity of the learning process: Through the interfaces of voices, pointing, mails, icons, even videos, the learner can communicate with experts, peers, or other materials effectively, in the form of synchronous or asynchronous communication. Hence, the expert is more reachable and the knowledge is more available.

- Situating of instructional activity: Via wireless applications, the learning could be embedded in daily life. The problems encountered, as well as the knowledge required are all presented in authentic context, which helps learners notice the features of problem situations that make particular actions relevant.

- Integration of instructional content: The wireless learning environment integrates many information resources, and supports learners to do non-linear, multidimensional, and flexible learning and thinking. It especially facilitates complex and ill-structured learning content, such as the cross-subject, themebased learning activities.

In the past 20 years, the constructivist paradigm has dominated educational research. From a constructivist perspective, the purpose of education is to cultivate independent and self-directed learners. Bruner's metaphor of scaffolding (Bruner, 1983) provides an explicit strategy to direct teaching toward this end. Scaffolding refers to the interactive support that instructors, or more skilful peers, offer learners to bridge the gap between their current skill levels and a desired skill level. In the process, the amount of support is gradually withdrawn as the learners become more proficient. Ultimately, the learners can complete tasks on their own (Greenfield, 1984). Therefore, the method of scaffolding consists of two major processes: one is 'support building', the other is 'support fading'. Cazden has used a vivid and familiar picture to make clear the concept and the pervasiveness of its exemplars. The literature review suggests that scaffolding can enhance comprehension, improve independent learning and application, and promote knowledge transfer (Bruner, 1983; Greenfield, 1984; Cazden, 1988). Evidence of these advantages has been found in many studies in the field of language and cognitive development (Boyle \& Peregoy, 1990; Gallimore et al., 1989; Smolucha \& Smolucha, 1989; Day \& Cordon, 1993). At the same time, limitations of scaffolding have also been pointed out. This technique has been criticised for its lack of discussion concerning development of the expert's role in providing the novice with assistance (Gaffney \& Anderson, 1991). Its implementation has also been criticised for not being able to capture the challenge of responding to the diversity of children's intentions in classroom teaching (Dyson, 1990).

This paper reports a wireless application designed to scaffold students learning about bird-watching. Based on that, the influence of this technology on the instructional process, management, and evaluation has been investigated. The aim is to construct an outdoor mobile learning activity supported with up-to-date wireless technology. The Bird-Watching Learning (BWL) system is implemented on a wireless mobile ad-hoc network. In the BWL system, each learner's device is a PDA (Personal Digital Assistant) with a Wi-Fi-based wireless LAN (IEEE 802.11b) card, to form an ad-hoc learning environment. The BWL system offers a mobile learning system which scaffolds the learner. The formative evaluation has the following two 
purposes: to explore the possible roles and scaffolding aids that the mobile learning device offers for bird-watching activities and to investigate whether student learning benefited from the mobility, portability, and individualisation of the learning device.

\section{Basic ideas and challenges}

The scaffolding technique (Jackson et al., 1996) has drawn a great deal of interest from educational technology researchers, because it can provide a more realistic learning environment with rich and varied support. In the area of educational research, the term 'scaffolding' has been used to describe various instructional techniques for supporting learning activities that reflect authentic task situations. Scaffolding enables the learner to engage in out-of-reach activities; having a 'knowledgeable other' or 'more capable peer' to support the learner and 'share the cognitive load' (Jackson et al., 1996). The processing and integrating capabilities of computers can create a realistic, interactive, support-rich, and individualised learning environment. These characteristics make computers ideal for implementing scaffolding. Several researchers have developed scaffolding computer-based instruction, integrating more than one medium to support learners' knowledge construction in authentic learning activities (Steiner \& Moher, 1994; Jackson et al., 1996). However, the discussion focused mainly on the software development and the support-building models.

Lieberman \& Linn (1991) contend that scaffolding is one of several ways that computers can be used to encourage self-directed learning in students. The Cognition and Technology Group at Vanderbilt University (Bransford et al., 1993) raised the problem of over reliance on computer support and stress the need to educate individuals to be independent and active learners. Therefore, there is a need to emphasise the support-fading component of scaffolding. To use this skill, the major challenge is to determine when the support should be faded, and how much the support should be reduced. This requires assessment of the learner's mastery level at any point during scaffolding. With this notion, Day and Cordon (1993) developed a method of scaffolding using five levels of support, where they faded support in a sequence of authentic practices. The support consisted of a series of hints, ordered in terms of explicitness. Each practice was used as an assessment of the learner's mastery of the learning task. Based on the learner's performance of the current practice, the instructor decided if it was the right time to reduce the current support level in the next practice. Their study provided a good scaffolding model which can be used in scaffolding computer-based or integrated-media-based instruction. With the mass storage and dynamic ability of the computer, this model can be easily built, and quantitative evaluation data can be easily collected.

Using Day and Cordon's model, Kao and Lehman (1997) proposed the basic elements of scaffolded instruction which are especially relevant to integrated-media design. They provided the example of an adult supporting an infant learning to walk (Cazden, 1988) for illustration.

- Hierarchical component skills: It is the instructor's responsibility to decompose the final task into hierarchical components, based on the nature of the task and the learner's ability. In the example of an adult aiding an infant, the child must learn how to balance on his feet before taking his first step. The child then must learn how to balance on his first step before stretching out for the second.

- Decreasing support levels: In the sense of support withdrawal, the instructor 


\section{Y.S. Chen et al.}

must recognise what kind of support is crucial in the learning, and classify the support into decreasing levels. In the example of an adult aiding a toddler, the levels of support could be: helping by holding two hands, helping by holding one hand, helping by holding one finger, etc.

- Repetitive authentic practice: The instructor has to set up a sequence of authentic practices involving the performance of the same skills. In the walking example, the child actually experiences the practical task, walking. The practice of walking involves the same component skills, and the practice can be repeated in different realistic settings: walking on carpet, walking on solid ground, walking on sand, and so forth.

- Ongoing assessment: The instructor must measure the learner's progress against the global picture of the task, and make corrections when needed. In the walking example, the adult observes and measures the child's progress with each baby step. If holding one hand of the child doesn't keep his balance, the adult gives him the other hand right away. If the child walks well by holding one hand, the adult will consider letting him walk by holding only one finger.

An operational definition of scaffolding could be as follows. The instructor, or the more skilful peer decomposes the task into hierarchical subtasks, classifies the amount of support in decreasing levels, and sets up a repetitive authentic practice. The practice begins with the highest level of support and the lowest level of subtask. With the completion of each subtask in the practice, the instructor measures the learner's performance and judges the level of support he/she should provide and lets the learner perfect the component subtask that he/she can manage. The new technology of wireless applications even extends the abilities and territory of computers. The characteristics of interactivity, situating, and integration could facilitate the application of the four basic elements of scaffolding. It appears that the integration of scaffolding and wireless application will create an effective learning environment for future classrooms.

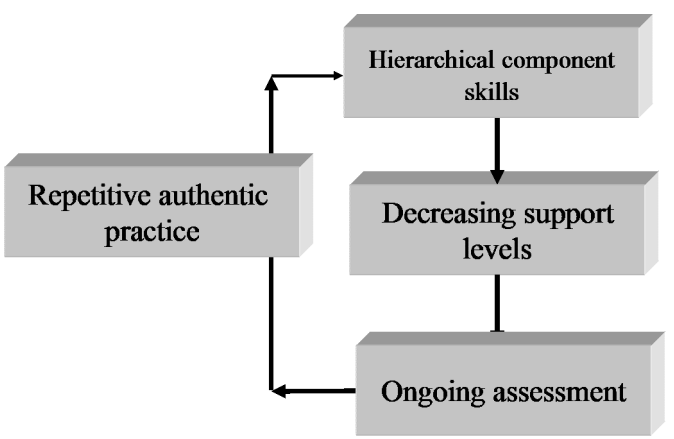

The mobile birdwatching system, the components of the scaffolding includes all four of the elements listed above. To implement the scaffolding system, a logical loop-structure was used to combine all of the components, as illustrated in Fig. 1. The key point of Fig. 1. The implementation structure of a scaffolding model scaffolding is to provide different levels of support, where the scaffolding level is determined according to the level of ability of each learner. With ongoing assessment, each learner's level of ability can be formally evaluated. The aim of scaffolding is to eliminate the need for assistance and decrease the level of support when the learner has increased his or her learning ability. During repetitive authentic practice, the system also provides hierarchical learning tools and skills to assist the learner to easily learn/accept the knowledge. 


\section{The BWL system}

The wireless mobile ad-hoc learning environment

The bird-watching activity was designed and built on a wireless mobile ad-hoc network (Johnson \& Maltz, 1996; Lin \& Liu, 1999; Chen \& Nahrstedt, 2000) which is described below. The wireless mobile ad-hoc networking environment is formed by several mobile wireless devices. Each learner has a mobile learning device, which is a handheld device (PDAs) with a Wi-Fi-based (IEEE 802.11b protocol) wireless network card. The instructor has a small-sized mobile notebook with a Wi-Fi-based wireless network card and a digital video camera. The learners and the instructor

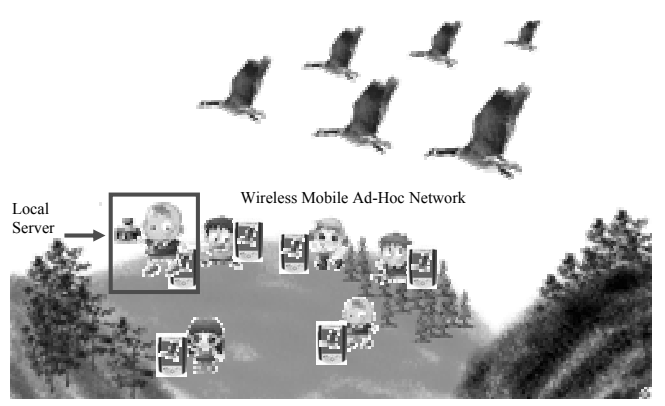

Fig. 2. The scenario of a bird-watching activity form a wireless mobile ad-hoc learning environment. The existing MANET (Mobile Ad-hoc NETwork) routing protocols (Johnson \& Maltz, 1996; Chen \& Lai, 2001; Chen et al. 2002) support the wireless transmission of the bird-picture/video and birdinformation between all of the learners' mobile learning devices. The scenario of a bird-watching activity is shown in Fig. 2.

The scaffolding-aid-based BWL system

Birding watching tours are usually offered by a local bird watching society and led by experts. They first look for the target bird and take a clear and close-up view of the bird through a high-quality telescope. Then, they let the learners take turns observing the bird through the telescope. In the meantime, they provides hints and guidance for identification of that particular bird. In the process, the knowledge and enthusiasm of the experts becomes a role model. Their vivid description of the bird usually arouses the learners' attention and interest. The hints and guidance provided scaffolds the learner's comprehension but the effect and level of the learning depends completely upon the expert. Besides, not every student gets the chance for a close observation through the high-quality telescope, as the bird might fly away while students are still waiting in line. It is also difficult for the expert to provide guidance to meet the needs of each learner, or to answer questions. Outdoor educational activities, such as this, benefit greatly from the aid of wireless technology.

The BWL system has the key characteristic of scaffolding depending on the student's capability. The BWL system offers a structured-assistance learning system; more assistance is given for beginners, and as they becomes more proficient, less assistance is given. The BWL system tries to provide a learning system with a different level of assistance. To know the students level of learning, the BWL system also contains a testing subsystem that evaluates each learner's level of learning. In this study, a learner is called an $m$-learner if the learner uses a mobile learning device to acquire and learn through a wireless transmission tool anytime and anywhere.

Implementation of hierarchical component skills. The BWL system uses a hierarchical component skills model. The component of the hierarchical component skills is divided into mobile bird-watching hardware and software. First, the mobile bird-watching hardware includes a handheld mobile learning device (PDA) 


\section{Y.S. Chen et al.}

containing an IEEE $802.11 \mathrm{~b}$ wireless network card. The instructor has an extra device, a notebook (a local server) and a digital video camera. All of these devices form a wireless mobile ad-hoc learning environment. It is a radically different learning environment compared to a conventional classroom. Knowledge acquisition is through a wireless handheld device, not the conventional book. The mobile bird-watching software includes the following interfaces.

- The wireless bird-watching interface: This interface includes $m$-instructor and $m$-learner interfaces. The $m$-instructor and $m$-learner interfaces are executed on the instructor's notebook and the $m$-learners' mobile devices, respectively. Using the $m$-instructor interface, bird pictures and video files can be broadcast to $m-$ learner interface by wireless transmission. This means that every student gets an equal chance for close observation through the $m$-learner interface.

- The wireless bird-searching interface: For hierarchically learning the birdknowledge, the BWL system offers a data-mining bird-searching system to assist the $m$-learner to easily search for the bird knowledge as illustrated in Fig. 3. The data-mining bird-searching system automatically produces bird query conditions, such as size and colour of the bird. By following the bird-searching operations

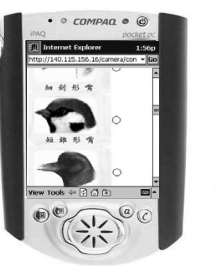

(a)

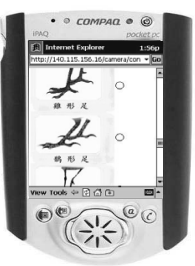

(b)

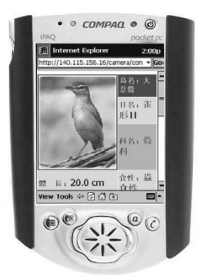

(c)

Fig. 3. The bird-watching and searching interfaces step-by-step, the $m$-learner will be able to acquire related bird knowledge from the instructor's notebook through the wireless transmission. The instructor's notebook contains a complete bird database of the birds in Taiwan. In addition the data-mining bird-searching system supports different levels of assistant functions by adjusting the difficulty-level of bird-query conditions, based on the ability of each individual $m$-learner. Using the wireless bird-searching interface, each $m$ learner can become familiar with bird ecology and any related knowledge on a step-by-step basis.

- The wireless bird-database interface: The wireless bird-database interface is built on the instructor's notebook. Each $m$-learner can dynamically query the bird knowledge of the database through the wireless handheld PDA. A web-based bird database is also provided on (http://140.115.155.87/twbd/new/mini.html). The BWL system builds a local bird-database interface in the instructor's notebook.

- The wireless bird-searching trace file: To understand the bird-searching patterns for each $m$-learner, a trace file is created in the instructor's notebook to record all bird-query actions during each bird-watching activity.

Implementation of repetitive authentic practice. To evaluate repetitive authentic practice, three tests, a pre-test, a middle-test (or $m$-learning), and a post-test, were used as shown in Fig. 4(a). The pre-test result assessed the learner's ability prior to the bird-watching activity. The post-test result assessed each learner's ability after the bird-watching activity. The middle-test was used to guide the students' attention during the bird-watching activity. That is, the questions in the middle-test were designed for guiding the students' attention to the key features of the bird, which were usually provided by the expert in the traditional bird-watching activity. 

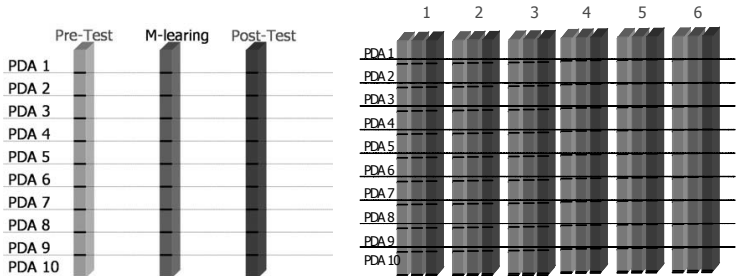

Fig. 4. (a) Three tests in a bird-watching activity

(b) Six bird-watching activities in a season

The test content will be described later. As illustrated in Fig. 4(b), the BWL system arranges six bird-watching activities throughout a season. This offers a long-term learning process and a learning history record is created.

Implementation of the ongoing assessment. In authentic practice, the teacher assists the students in accordance with their learning efficiency. Therefore, an ongoing assessment system was designed; this was divided into the following interfaces.

- The wireless bird-question assignment interface. This interface was designed for instructors. There are a huge number of bird records in the bird-database (http://140.115.155.87/twbd/new/mini.html). Therefore, the wireless bird-question assignment interface provides a filtering operation to filter bird records from the bird-database (there are about 535 Taiwan bird records). It filters the records according to month (from January to December), altitude (low, middle, high), and ecological environment (water habitat, forest, wide area). As illustrated in Fig. 5(a), 535 Taiwan bird records may be filtered to 35 Taiwan bird records. Finally, as illustrated in Fig. 5(a) and Fig. 5(b), the instructor selected 10 birdquestions from the filtered bird records, and transmitted them to the wireless bird-answer interface. It was used by all $m$-learners for the pre-test, the middletest, and the post-test.

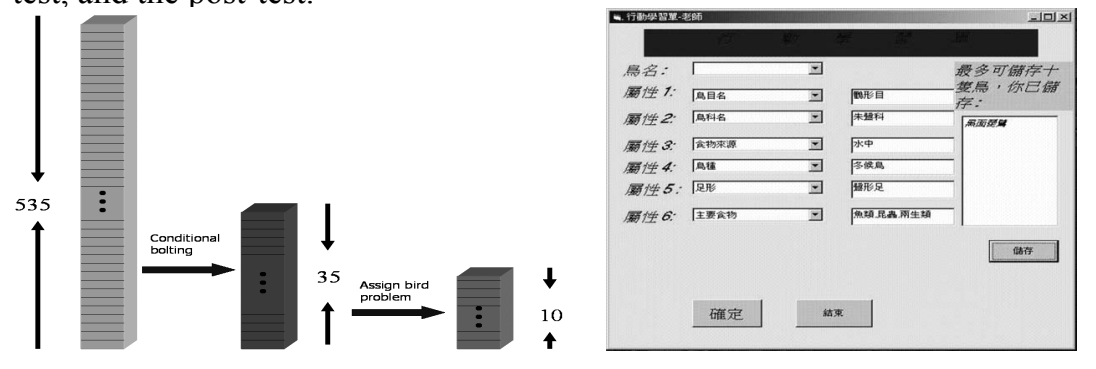

Fig. 5. (a) The wireless bird-question assignment interface (b) the bird-question assignment interface

- The wireless bird-answer interface. The wireless bird-answer interface uses a pull-down window-interface as shown in Fig. 6(a). The pull-down window-

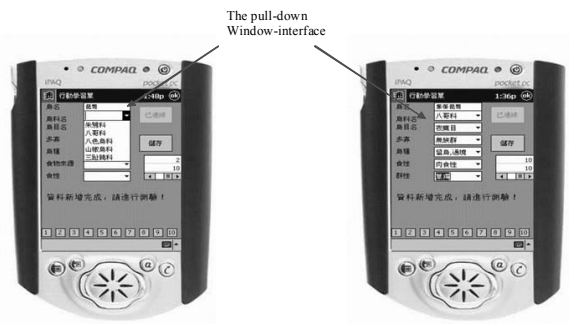

Fig. 6. The wireless bird-answer interface interface offers a user-friendly input interface for $m$-learners, as illustrated in Fig. 6(b). All input-data under the birdanswer interface for all $m$ learners were transmitted wireless to the instructor's device to compile a statistics table. 
- The learn-statistics interface. During the bird-watching activity, all answer records were collected into the instructor's notebook. Consequently, a statistics interface was designed for the instructor's notebook, as displayed in Fig. 7. The
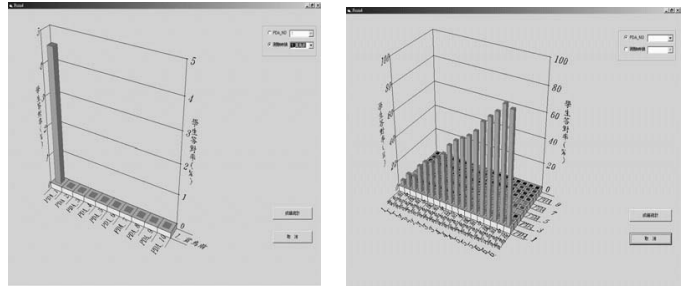

Fig. 7. The learn-statistics interface system was implemented by two kinds of programs. The client program runs on the PDA by using RDA (remote data access), and the server program runs on the notebook using the SQL 2000.

Implementation of decreasing support levels. The decreasing levels of support was implemented by adjusting the number of selected-items in the pull-down window based menu. The system provides different levels of assistance because each $m$ -

The number of selected-item

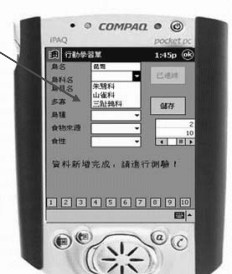

(1)

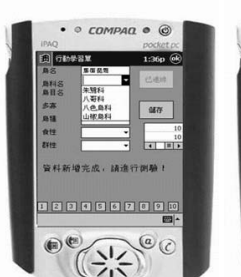

स:

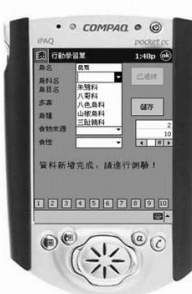

Fig. 8. Example of decreasing support levels learner requires different levels of support. For each $m$-learner, the selected number of items is three if the average score is less than $40 \%$ (see Fig. 8a). When the average score of the $m$-learner becomes higher, the number of selected-items is increased. For other example, when the average score of the $m$-learner is higher than $40 \%$ and lower than $60 \%$, the BWL system provides four selected-items (see Fig. $8 \mathrm{~b}$ ). In addition, if the average score of the $m$-learner is higher than $60 \%$, the BWL system provides five items as illustrated in Fig. 8c.

\section{Evaluation}

\section{Research design}

With the help of the BWL system, learning through the outdoor activity of birdwatching (described earlier) can be more independent and self-reliant. It creates the fair opportunities and experiences necessary (observing a bird close-up) for students to develop the knowledge and skills needed to make responsible decisions (identifying bird features) and to take actions (searching for relevant information) dealing with their own learning.

Because the BWL system was first introduced to the traditional outdoor birdwatching activities, three phases of experiments were designed based on the role of BWL in learning, as well as the level of BWL integration in the bird-watching activities. The intention of the three-phase design of the experiments was to scaffold the learners' ability and independence by shifting the task responsibility from the expert to the learners with the BWL program.

- Phase 1: BWL as a tool for enhancement. The BWL procedure was applied at the end of a traditional bird watching process. That is to say, the bird watching 
process was lead by the expert. The expert confirmed the name and key features of the bird being observed directly. At the end of the activity, the learners were gathered together and worked in teams. Each team used the key features of the bird in question to search for the relevant information with the PDA. They then could match their results with the expert's previous description.

- Phase 2: BWL as a tool for discovery. The BWL procedure was applied at the beginning of the bird watching process. In the meantime, the expert only provided hints and led the learners to notice the key features of the bird. Then, the learners assembled in teams and used the features of the bird they identified to search for the name and the relevant information by means of the PDA. This way, all the learners had the chance to fully observe, explore and discuss. After each group reached an answer, the expert gathered the learners for debriefing and further guidance.

- Phase 3: BWL as a tool for independence. The BWL procedure was applied to the whole bird-watching process. The PDA completely took over the role of the expert. The learners could only observe the static image of the bird on the PDA. They had to identify the key features of the bird on their own, to guide them in their search later. And, the search result was the only information they could learn about the bird. During the entire process, the teacher only checked their results for correction.

Along with the three-phase design of experiments, a control group was used to evaluate the benefits of BWL with regards to learning. The students in the control group used the guidebook to get the relevant information of the bird being observed as per the traditional bird-watching activity.

\section{Participants}

Three elementary schools were chosen to participate in the study from more than 20 competitors. They were Chang-Hsing at Keelung, Ming-Tao at Taipei, and Hsi-Men at Tainan, located near three of the 10 best bird watching sites in Taiwan. The

Table 1. Numbers of teams in each group for each school

\begin{tabular}{lccc}
\hline School & Participants & Teams in BWL & Teams in Guidebook \\
\hline Chang-Hsing & 30 4th graders & 7 & 8 \\
Ming-Tao & 28 5th graders & 7 & 7 \\
Hsi-Men & 28 6th graders & 7 & 7 \\
Totals & 86 & 21 & 22 \\
\hline
\end{tabular}
students at each school were randomly assigned into two groups, the BWLassisted group and the guidebook-assisted group. Each group

was lead by a natural sciences teacher in the school of their school, and a bird guide (i.e. the expert) from the local wild life society. The students worked in pairs. Table 1 lists the numbers of teams or pairs in each group for three schools.

\section{Instruments}

- Pre-test. Before the activity, the teacher and the expert devised a list of 10 kinds of birds that might be observed for the pre-test. For each bird, each team in either BWL or guidebook group had to answer six multiple-choice questions on features of the bird. The pre-test was administered in the form of an electronic test on the PDA.

- Worksheet. According to the features of the bird being observed in the activity (at least six birds per activity), six multiple-choice questions were assigned on the 


\section{Y.S. Chen et al.}

worksheet. Instead of measuring the learners' ability, the questions were designed to guide the students' attention to the key features of the bird, which is usually provided by the expert in the traditional bird-watching activity. To answer those questions, the learners had to identify the bird's features and search for the relevant information with either BWL or guidebook. The team in the BWL group received the questions and provided their answers using the electronic worksheet on the PDA. On the other hand, the team in the guidebook group received the questions orally from the expert and provided answers on a paper worksheet.

- Post-test. The post-test was administered to all the teams in both groups after the activity was completed. It was the same as the pre-test, except it only involved the birds being observed in the activity (at least six birds per activity).

\section{Procedure}

Each participant school conducted six bird watching activities in the autumn semester of 2002. That is, two activities were conducted in each of the three phases of the experiments. Although the timing for applying the BWL/guidebook-assisted procedure was different for each phase, each activity consisted mainly of the following three components.

- Pre-test. The pre-test was administrated right before the activity, either before departure or on the bus on the way to the bird-watching sites.

- The BWL/guidebook-assisted procedure. In the activity, the teacher and the expert in both groups had the following equipment: a notebook; a telescope with tripod; and a digital camera with telephoto lens or a camera connector to the telescope. The team in the BWL group was given a PDA. The team in the guidebook group was given a two-cone telescope and a copy of the guidebook. The experts in both groups first targeted the same kind of bird with the telescope (at least six birds per activity). A digital picture of the bird was taken and transferred to the notebook. Worksheet questions were further devised according to the key features of the bird. The team in the BWL group acquired the picture and worksheet questions, identified the bird features, searched for the relevant information, and answered the questions with BWL. The team in the guidebook group identified the bird features with the two-cone telescope, searched for the relevant formation with guidebook, received the questions orally, and provided answers on a paper worksheet. Finally, the teachers in both groups checked the students' answers.

- Post-test. After the activity, the post-test was administered to the learners either on the return trip or right after being back to the school in their respective class rooms.

\section{Results}

Table 2 presents the means and the standard deviations of the pre-test and the posttest for each activity.

For Activity 1, only one school's pre-test data was collected, and no significant group difference was found in the post-test $(t=-0.469, p>0.05)$. For the rest of the activities, ANCOVA was used to test the group difference on post-test with pre-test as covariate (see Table 3). Significant group differences were found on activities 3, 4, 5 and 6. Figure 9 further presents the progress curves of post-test mean scores for activities of both groups. 
Table 2. Means and standard deviations of pre-test and post-test for each activity

\begin{tabular}{lrrrr}
\hline Test & \multicolumn{2}{c}{ BWL } & \multicolumn{2}{c}{ Guidebook } \\
& mean & s.d. & mean & s.d. \\
\hline Phase one & & & & \\
Activity 1 Pre $^{\mathrm{a}}$ & 47.43 & 3.78 & 39.71 & 8.60 \\
Activity 1 Post $^{\mathrm{b}}$ & 47.00 & 20.95 & 50.14 & 16.40 \\
Activity 2 Pre & 51.65 & 11.39 & 50.67 & 11.38 \\
Activity 2 Post & 52.45 & 14.31 & 53.45 & 11.18 \\
Phase two & & & & \\
Activity 3 Pre & 64.10 & 16.08 & 52.62 & 13.98 \\
Activity 3 Post & 65.86 & 16.99 & 48.38 & 19.61 \\
Activity 4 Pre & 53.00 & 12.48 & 47.25 & 13.78 \\
Activity 4 Post & 69.65 & 22.97 & 51.62 & 14.28 \\
Phase three & & & & \\
Activity 5 Pre & 64.95 & 19.84 & 55.50 & 15.97 \\
Activity 5 Post & 81.67 & 12.90 & 64.45 & 20.13 \\
Activity 6 Pre & 65.33 & 20.31 & 51.68 & 18.50 \\
Activity 6 Post & 74.29 & 11.39 & 50.95 & 17.84 \\
\hline
\end{tabular}

${ }^{a}$ For activity 1 pre-test, data from Chang-Hsing and Ming-Tao were not complete.

${ }^{b}$ For activity 1 post-test, the guidebook group data from Hsi-Men were not complete.

Table 3 AnCOVA for post-test of each activity

\begin{tabular}{clll}
\hline Phase & Source & d.f. & F \\
\hline $\mathbf{1}$ & Activity 2 Post & & \\
& Activity 2 Pre & 1 & 3.613 \\
& Group & 1 & 0.042 \\
\hline \multirow{2}{*}{ Activity 3 Post } & & \\
& Activity 3 Pre & 1 & 1.359 \\
& Group & 1 & $5.343^{*}$ \\
& Activity 4 Post & & \\
$\quad$ Activity 4 Pre & 1 & 1.216 \\
& Group & 1 & $8.515^{* *}$ \\
\hline \multirow{3}{*}{ Activity 5 Post } & & \\
& Activity 5 Pre & 1 & $8.354^{* *}$ \\
& Group & 1 & $7.250^{* *}$ \\
& Activity 6 Post & & $5.977^{* *}$ \\
Activity 6 Pre & 1 & $17.950^{* *}$ \\
\hline & Group & 1 &
\end{tabular}

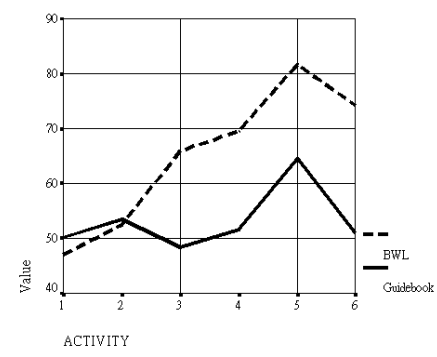

Fig. 9. The progress curves of the post-test means for the activities of both groups

$$
\begin{gathered}
\text { Activity } 1 \& 2 \text { for Phase } 1 ; \\
\text { Activity } 3 \& 4 \text { for Phase } 2 \text {; } \\
\text { Activity } 5 \text { for Phase } 3
\end{gathered}
$$

$* \mathrm{p}<0.05 . * * \mathrm{p}<0.01$

For the activities in Phase $2 \& 3$, significant differences were found between the groups for Activity $3(F=5.343, p<0.05)$, Activity $4(F=8.515, p<0.01)$, Activity $5(F=7.250, p<0.01)$ and Activity $5(F=17.950, p<0.01)$ with the BWL group outperforming the guidebook group. This means that the learners benefited substantially from BWL, even though the expert support was gradually withdrawn. The data from phase two provides evidence that the BWL system could be an effective discovery tool for bird identification. Although the learners had to search for the relevant information (with either BWL or guidebook) without any help from the expert in phase three, both group got their highest mean scores on Activity 5. The possible explanation is that the learners might have better perceived their own responsibility for completing the task and gave more attention to their performance in this activity (i.e. the Hawthorne effect). The effect of Phase 3 to learning was therefore increased beyond what might be expected under normal conditions. This 
possible effect did hold for the second activity of Phase 3. The Activity 6 data reflected their normal performance under the condition of Phase 3. It was found that the BWL group performed even better than in the other two phases. This was because the learners had to spend more mental effort for completing the task on their own. In contrast, the learners in the guidebook group almost maintained at a fixed level of performance in all three phase conditions, except for Activity 5. It is possible that the learners' mastery level increased as the support level decreased phase by phase and hence the effects just balanced out.

\section{Conclusion}

This paper has reported upon a developed a mobile scaffolding-aid system for birdwatching. The proposed Bird-Watching Learning (BWL) system was designed using a wireless mobile ad-hoc network. In the BWL system, each learner's device had a PDA (Personal Digital Assistant) with Wi-Fi-based (IEEE 802.11b) wireless network card. The BWL system was specifically developed to integrate the scaffolding model with the BWL system. Three elementary schools in Taiwan used the BWL system for bird-watching. A formative evaluation from their experiences was undertaken. Based on these findings, it was found that the children who used the bird watching system improved their learning, above and beyond what would normally be expected they would learn.

\section{Acknowledgement}

This work was supported by learning technology, sponsored by the Ministry of Education, Taiwan: 90 A-H-FA07-1-4 and 91 A-H-FA07-1-4.

\section{References}

Boyle, O.F. \& Peregoy, S.F. (1990) Literacy scaffolds: Strategies for first- and secondlanguage readers and writers. Reading Teacher, 44, 3, 194-200.

Bransford, J., Goin, L., Goldman, S., Hasselbring, T., Pellegrino, J., Sharp, D. \& Vye, N. (1993) Integrated media: Toward a theoretical framework for utilizing their potential. Journal of Special Education Technology, 12, 71-85.

Bruner, J. (1983) Child's talk. Learning to Use Language. W.W. Norton, New York.

Cazden, C.B. (1988) Classroom discourse. The Language of Teaching and Learning. Heinemann, Portsmouth, NH.

Chen, Y-S., Chen, T-S. \& Huang, C-J. (2002) SOM. Spiral-Fat-Tree-Based on-Demand Multicast Protocol in a Wireless Ad-Hoc Network. Computer Communications, 25, 17, 1684-1695.

Chen, Y-S. \& Lai, K.-C. (2001) MESH: Multi-Eye Spiral-Hopping Protocol in a Wireless Ad-Hoc Network. IEICE Transactions on Communications, E84-B, 8, 2237-2248.

Chen, S. \& Nahrstedt, K. (2000) Distributed Quality-of-Service Routing in Ad Hoc Networks. IEEE Journal on Selected Areas in Communications, 17, 8, 1594-1603.

Day, J.D. \& Cordon, L.A. (1993) Static and dynamic measures of ability: An experimental comparison. Journal of Educational Psychology, 85, 75-82.

Dyson, A.H. (1990) Weaving possibilities: Rethinking metaphors for early literacy development. Reading Teacher, 44, 3, 202-213.

Gaffney, J.S. \& Anderson, R.C. (1991) Two-tiered scaffolding: Congruent processes of teaching and learning. In Literacy for a Diverse Society: Perspectives, Programs and Policies. (ed. E.H. Hiebert) pp. 184-198, Teachers College Press, New York. 
Gallimore, R., Tharp, R. \& Rueda, R. (1989) The social context of cognitive functioning in the lives of mildly handicapped persons. In Cognitive Approaches in Special Education (ed. D.A. Sugden) pp. 51-82. Falmer Press, Lewes.

Greenfield, P.M. (1984) A theory of the teacher in the learning activities of everyday life. In Everyday Cognition (eds. B. Rogoff \& J. Lave) pp. 117-138. Harvard University Press, Cambridge, MA.

Jackson, S.L., Stratford, S.J., Krajcik, J. \& Soloway, E. (1996) Model-It: A Case Study of Learner-centered Software Design for Supporting Model Building. Report No. MVK50700. Michigan University, Ann Arbor, MI. (ERIC Document Reproduction Service No. ED 446903)

Johnson, D.B. \& Maltz, D.A. (1996) Dynamic Source Routing in Ad Hoc Wireless Networks. In Mobile Computing (eds T. Imielinski \& H. Korth) pp. 81-153. Kluwer Academic Publishers, Norwell, Mass.

Kao, M.T. \& Lehman, J.D. (1997) Scaffolding in a computer-based constructivist environment for teaching statistics to college learners. Paper presented at the 1997 Annual Meeting of American Educational Research Association, Chicago, IL. (ERIC Document Reproduction Service No. ED 408317)

Lieberman, D.A. \& Linn, M.C. (1991) Learning to learn revisited: Computers and the development of self-directed learning skills. Journal of Research on Computing in Education, 23, 3, 373-395.

Lin, C.R. \& Liu, J.S. (1999) QoS Routing in Ad Hoc Wireless Networks. IEEE Journal on Selected Areas in Communications, 17, 8, 1426-1438.

Smolucha, L. \& Smolucha, F. (1989) A Vygotskian perspective on critical thinking. Paper presented at the conference on Science and Technology for Education in the 1990s: Soviet and American Perspectives, Meadville, PA. (ERIC Document Reproduction Service No. ED 314770)

Steiner, K.E. \& Moher, T.G. (1994) Scaffolding story construction with interactive multimedia. Journal of Educational Multimedia and Hypermedia, 3, 173-195.

\section{Notice: \\ Due to SARS problems, the "Second IEEE International Workshop on Wireless and Mobile Technologies in Education" has been postponed by three months.}

The new details are as follows:

Second IEEE International Workshop on

Wireless and Mobile Technologies in Education (WMTE 2003)

National Central University, JungLi, Taiwan

March 23-25, 2004

Submissions due:

September 15, 2003

Notification of acceptance:

October 31, 2003

Final articles due:

December 1, 2003

Workshop:

March 23-25, 2004

Proceedings to be published by IEEE Computer Society Press.

For further details, please check the website:

http://lttf.ieee.org/wmte2003/ 